\title{
Applications Of Mems In Robotics And Bio Mems Using Psoc With Metal Detector \& Wireless Image \& Voice Transmission
}

\author{
Azmeera Srinivas ${ }^{1}$, K.Satyavathi ${ }^{2}$ \\ ${ }^{1}$ Student, ${ }^{2}$ Associate Professor \\ ${ }^{1}$ Ece Department, Mallareddy Institute Of Engineering And Technology. \\ ${ }^{2}$ hod, Ece Department, Mallareddy Institute Of Engineering And Technology. \\ Hyderabad, Ap, India. ${ }^{1}$, Hyderabad, Ap., India ${ }^{2}$.
}

\begin{abstract}
This project deals with accelerometercontrolled robot with wireless image and voice transmission as well as metal Detector. This robot is prototype for the "Path Finder". This robot is controlled based on PSoCdevice using MEMS accelerometer remote. This can be moved forward and reverse direction using geared motors of 60RPM. Also this robot can take sharp turnings towards left and right directions. A high sensitive induction type metal detector is designed using colpitts oscillator principle and fixed to this robot. Also a wireless camera with voice is interfaced to the kit. When the robot is moving on a surface, the system produces a beep sound when metal is detected. This beep sound will be transmitted to remote place. Simultaneously the images around the robot will be transmitted to remote place. User can monitor the images and metal detection alarms on Television.
\end{abstract}

Keywords: PSoC designer 1.0, keil -c,PSOC device (CY8C29466), AT89S52.

\section{INTRODUCTION}

Embedded System is a combination of computer hardware and software, and perhaps additional mechanical or other parts, designed to perform a specific function, an embedded system. Each of these devices contains a processor and software and is designed to perform a specific function; the first such systems could not possibly have appeared before 1971. That was the year Intel introduced the world's first microprocessor. This chip, the 4004, was designed for use in a line of business calculators produced by the Japanese Company Busicom. In 1969, Busicom asked Intel to design a set of custom integrated circuits-one for each of their new calculator models. The 4004 was Intel's response rather than design custom hardware for each calculator, Intel proposed a general-purpose circuit that could be used throughout the entire line of calculators. Intel's idea was that the software would give each calculator its unique set of features.

The microcontroller was an overnight success, and its use increased steadily over the next decade. Early embedded applications included unmanned space probes, computerized traffic lights, and aircraft flight control systems. In the 1980s, embedded systems quietly rode the waves of the microcomputer age and brought microprocessors into every part of our kitchens (bread machines, food processors, and microwave ovens), living rooms (televisions, stereos, and remote controls), and workplaces (fax machines, pagers, laser printers, cash registers, and credit card readers).

It seems inevitable that the number of embedded systems will continue to increase rapidly. Already there are promising new embedded devices that have enormous market potential; light switches and thermostats that can be central computer, intelligent air-bag systems that don't inflate when children or small adults are present, pal-sized electronic organizers and personal digital assistants (PDAs), digital cameras, and dashboard navigation systems. Clearly, individuals who possess the skills and desire to design the next generation of embedded systems will be in demand for quite some time.

\section{System Design Model}

\section{A. Software design module}

The development process for the PSoC device differs from that of a traditional fixed function microprocessor. The configurable analog and digital hardware blocks give the PSoC architecture a unique flexibility that pays dividends in managing specification change during development and by lowering inventory costs. These configurable resources, called PSoC Blocks, have the ability to implement a wide variety of userselectable functions.

Each block has several registers that determine its function and connectivity to other blocks, multiplexers, buses, and to the IO pins. Iterative development cycles permit you to adapt the hardware as well as the software. This substantially lowers the risk of having to select a different part to meet the final design requirements. To speed the development process, the PSoC Designer Integrated Development Environment 
(IDE) provides a library of pre-built, pre-tested hardware peripheral functions, called "User Modules." User modules make selecting and implementing peripheral devices simple, and come in analog, digital, and mixed signal varieties. The standard User Module library contains over 50 common peripherals such as ADCs, DACs Timers, Counters, UARTs, and other not-so common peripherals Such as DTMF Generators and Bi-Quad analog filter sections. Each user module establishes the basic register settings that implement the selected function. It also provides parameters that allow you to tailor its precise configuration to your particular application.

For example, a Pulse Width Modulator User Module configures one or more digital PSoC blocks, one for each 8 bits of resolution. The user module parameters permit you to establish the pulse width and duty cycle. User modules also provide tested software to cut your development time. The user module application programming interface (API) provides high-level functions to control and respond to hardware events at runtime.

The development process starts when you open a new project and bring up the Device Editor, a graphical user interface (GUI) for configuring the hardware. You pick the user modules you need for your project and map them onto the PSoC blocks with point-and-click simplicity. Next, you build signal chains by interconnecting user modules to each other and the IO pins. At this stage, you also configure the clock source connections and enter parameter values directly or by selecting values from drop-down menus.

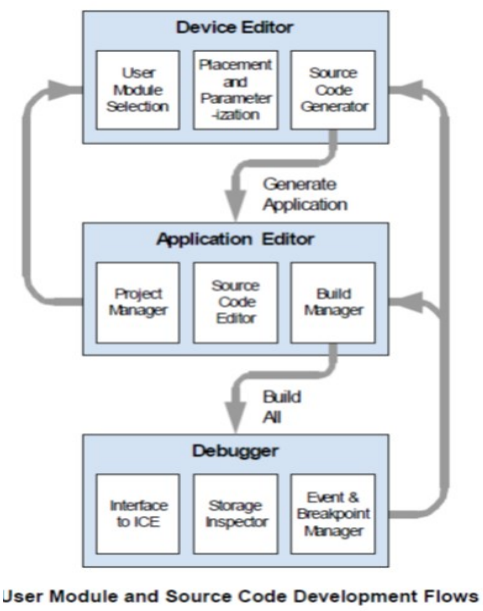

The last step in the development process takes place inside the PSoC Designer's Debugger subsystem. The Debugger downloads the HEX image to the In-Circuit Emulator (ICE) where it runs at full speed. Debugger capabilities rival those of systems costing many times more. In addition to traditional single-step, run-tobreakpoint and watch-variable features, the Debugger provides a large trace buffer and allows you define complex breakpoint events that include monitoring address and data bus values, memory locations and external signals.

\section{B. Hardware design module BLOCK DIAGRAM}

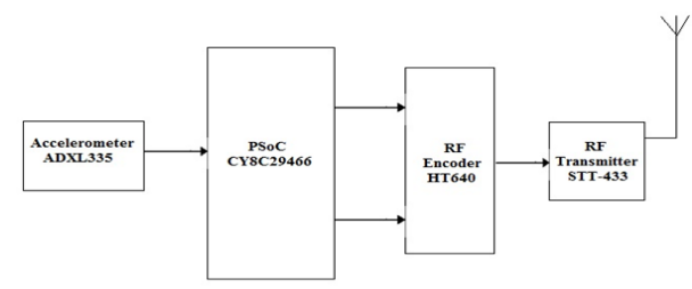

Figure: transmitter side of hardware implementation

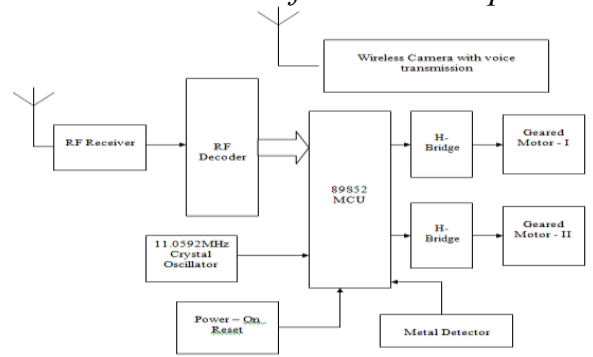

Figure: receiver side of the hardware implementation. 
DEMO CIRCUIT: Transmission Circuit

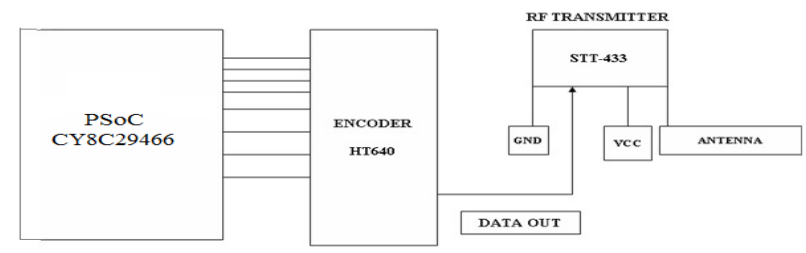

Figure: RF Transmission Circuit

The data sent from the PSoC is encoded and sent to RF transmitter. The data is transmitted on the antenna pin. Thus, this data should be received on the destination i.e, on RF receiver.

DEMO CIRCUIT: Reception circuit

The data transmitted into the air is received by the receiver. The received data is taken from the data line of the receiver and is fed to the decoder. The output of decoder is given to microcontroller and then data is processed according to the applications.

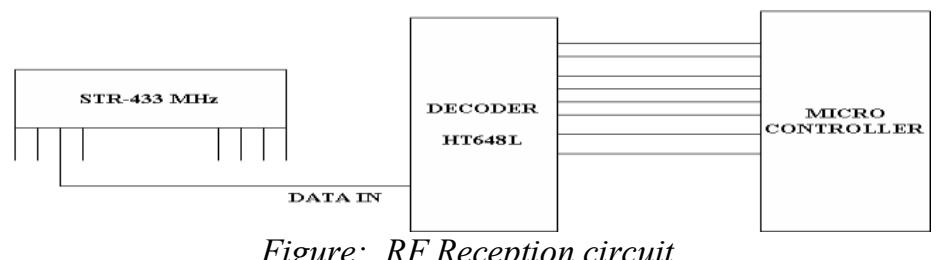

Figure: RF Reception circuit.

\section{i. ACCELEROMETERGENERAL DESCRIPTION}

The ADXL335 is a small, thin, low power, complete 3 -axis accelerometer with signal conditioned voltage outputs. The product measures acceleration with a minimum full-scale range of $\pm 3 \mathrm{~g}$. It can measure the static acceleration of gravity in tilt-sensing applications, as well as dynamic acceleration resulting from motion, shock, or vibration.

The user selects the bandwidth of the accelerometer using the CX, CY, and CZ capacitors at the XOUT, YOUT, and ZOUT pins. Bandwidths can be selected to suit the application, with a range of $0.5 \mathrm{~Hz}$ to $1600 \mathrm{~Hz}$ for the $\mathrm{X}$ and $\mathrm{Y}$ axes, and a range of $0.5 \mathrm{~Hz}$ to $550 \mathrm{~Hz}$ for the $\mathrm{Z}$ axis.

The ADXL335 is available in a small, low profile, $4 \mathrm{~mm} \times 4 \mathrm{~mm} \times 1.45 \mathrm{~mm}$, 16-lead, plastic lead frame chip scale package (LFCSP_LQ).

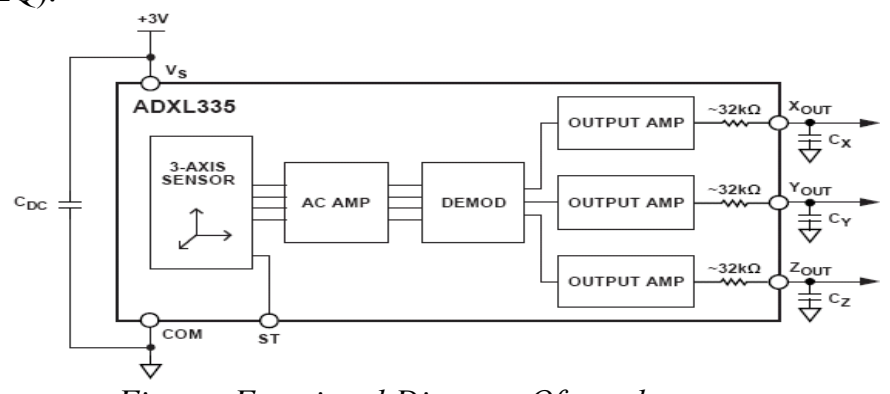

Figure: Functional Diagram Of accelerometer

\section{ii. METAL DETECTOR}

Metal detectors use electromagnetic induction to detect metal. Metal detector can help you to find the metals buried deep in the ground. Uses include de-mining (the detection of land mines), the detection of weapons such as knives and guns, especially at airports, geophysical prospecting, archaeology and treasure hunting. Metal detectors are also used to detect foreign bodies in food, and in the construction industry to detect steel reinforcing bars in concrete and pipes and wires buried in walls and floors. The simplest form of a metal detector consists of an oscillator producing an alternating current that passes through a coil producing an alternating magnetic field. If a piece of electrically conductive metal is close to the coil, eddy currents will be induced in the metal, and this produces an alternating magnetic field of its own.

\section{iii. WIRELESS CAMERA WITH VOICE TRANSMISSION}

A portable small-sized camera has a case having a ball-point pen appearance in a portion thereof and a through hole in one side, and a camera circuit part built in the case and for photographing an object through the through hole. The portable small-sized camera has the ball-point pen appearance, photographing a particular location in secret is possible without exposure to others. The camera circuit part is connected to a wireless transmission device for outputting a signal by a cable. A wireless receiving device at a remote location from the 
wireless transmission device receives a signal of the wireless transmission device for outputting or recording.The portable camera further includes a microphone and the transmissiondevice transmits a voice signal.

\section{Experimental Results}

This robot is prototype for the "Path Finder". This robot is controlled based on PSoC device using MEMS accelerometer remote with wireless image and voice transmission as well as metal Detector. This robot is controlled by accelerometer remote. A high sensitive induction type metal detector is designed using colpitts oscillator principle and fixed to this robot. Also a wireless camera with voice is interfaced to the kit.
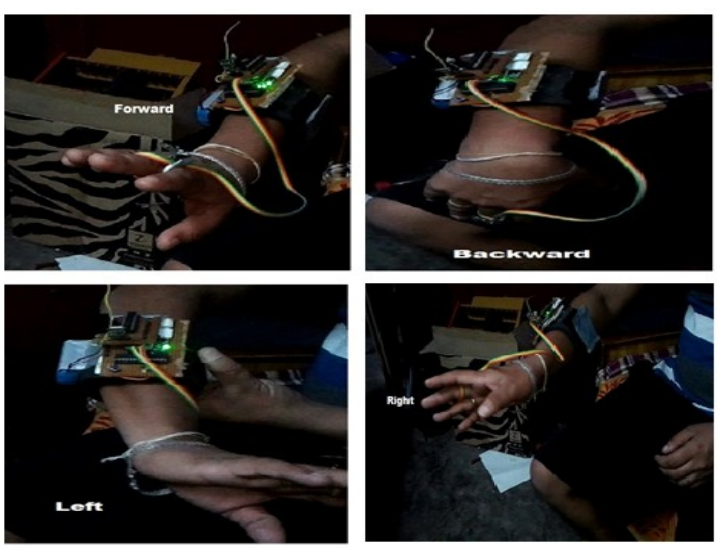

Figure: experimental set-up for four directions.

This can be moved forward and reverse direction using geared motors of 60RPM. Also this robot can take sharp turnings towards left and right directions.
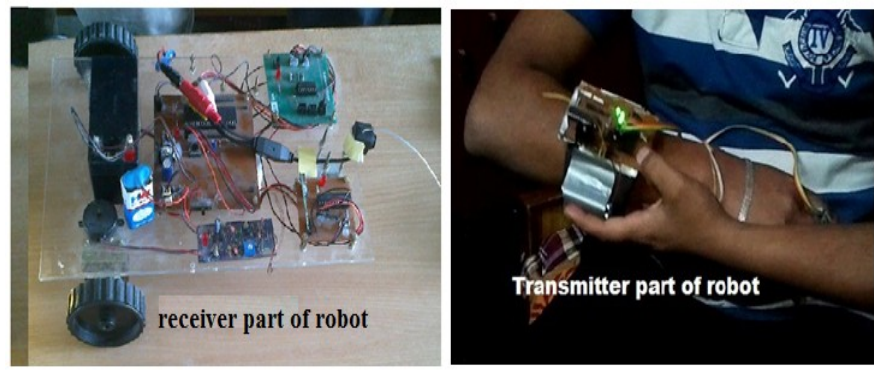

Figure: receiver and transmitter parts of the robot.

\section{Conclusion}

Embedded Systems plays a vital role in our day today life. They are used for household appliances like microwave oven to the satellite applications. They provide good man to machine interface. Automation is the further step in the world of Embedded Systems, which includes the elimination of the human being in the mundane applications. They are cost effective, accurate and can work in any conditions and round the clock.

\section{References}

[1]. Target Estimation for a Mine Detector with Wireless Image and Sound Transmission for Robot.

[2]. www.howstuffworks.com

[3]. www. Electronics for you.com

[4]. www. Electrikindia.com

[5]. Embedded system BY RAJ KAMAL

[6]. 8051 Microcontroller and embedded systems BY MAZZIDI. 